\title{
The location and function of respiratory fibres in the second cervical spinal cord segment: respiratory dysfunction syndrome after cervical cordotomy
}

\author{
Juan Lahuerta, Peter Buxton, Sam Lipton, David Bowsher
}

\begin{abstract}
After high cervical percutaneous cordotomy for pain in malignant disease, 12 patients died during sleep at postoperative intervals between 1 and 8 days. Nine died after a first cordotomy and three after a second (contralateral) procedure. All except one had known pulmonary disease before operation. The operated segment of the spinal cord (C2) was studied histologically after death. Superposition of lesion outlines made it possible to determine those parts of the lesioned areas common to all unilateral and bilateral cases respectively. All cases dying of presumed respiratory dysfunction syndrome had lesions involving the region of the anterolateral funiculus in the C2 segment containing "pain" fibres activated from the second to fifth thoracic dermatomes. The fibres whose destruction appeared to be responsible for respiratory dysfunction syndrome were completely intermingled with ascending "pain" fibres. The possibility of these fibres being afferent in function is discussed.
\end{abstract}

( $\Im$ Neurol Neurosurg Psychiatry 1992;55:1142-1145)

Respiratory dysfunction has been recognised as a potentially fatal complication of cervical cordotomy since the operation was first introduced. ${ }^{1-3}$ The condition, characterised by prolonged periods of apnoea, particularly during sleep, is sometimes referred to as "Ondine's Curse". Although more common after a bilateral procedure, it also occurs following unilateral cordotomy, and is as much to be feared after percutaneous as after open operation. ${ }^{45}$

Post-cordotomy respiratory dysfunction syndrome has been explained in terms of disruption of descending pathways controlling motor respiratory function. The nature of the responsible fibres which are anatomically interrupted in the anterolateral funiculus of the cervical spinal cord has been debated.

We studied the anatomical pathology of 38 cordotomies in 30 patients who died following percutaneous cervical cordotomy performed by us. ${ }^{6}$ We report the results of anatomoclinical correlations in 12 cases who died of presumed respiratory dysfunction syndrome.

\section{Material and methods}

Nine patients died within one to four days after unilateral cordotomy, and three within two to eight days after a second cordotomy. (table)

The spinal cords were removed into $10 \%$ formalin within 36 hours of death. Blocks were cut from the cervical cord (C1-C2) and included in paraffin, and sections cut at 3 or $4 \mu \mathrm{m}$ for staining by haematoxylin and eosin or cresyl violet (Nissl), and at $15 \mu \mathrm{m}$ for staining by the Heidenhahn method for myelin or by the Luxol Fast Blue method. Photographs were taken of those sections containing the most extensive lesions. The extent of the latter was determined by planimetry with a milli-

Table Patients dying of respiratory dysfunction syndrome following percutaneous cervical cordotomy

Centre for Pain Relief and Pain Research Institute, Walton Hospital, Liverpool, UK

J Lahuerta

P Buxton

S Lipton

D Bowsher

Correspondence to: Dr Bowsher, Pain Research Institute, Walton Hospital, Rice Lane, Liverpool L9 IAE, UK

Received 4 March 1991 and in final revised form 17 January 1992. Accepted 30 January 1992

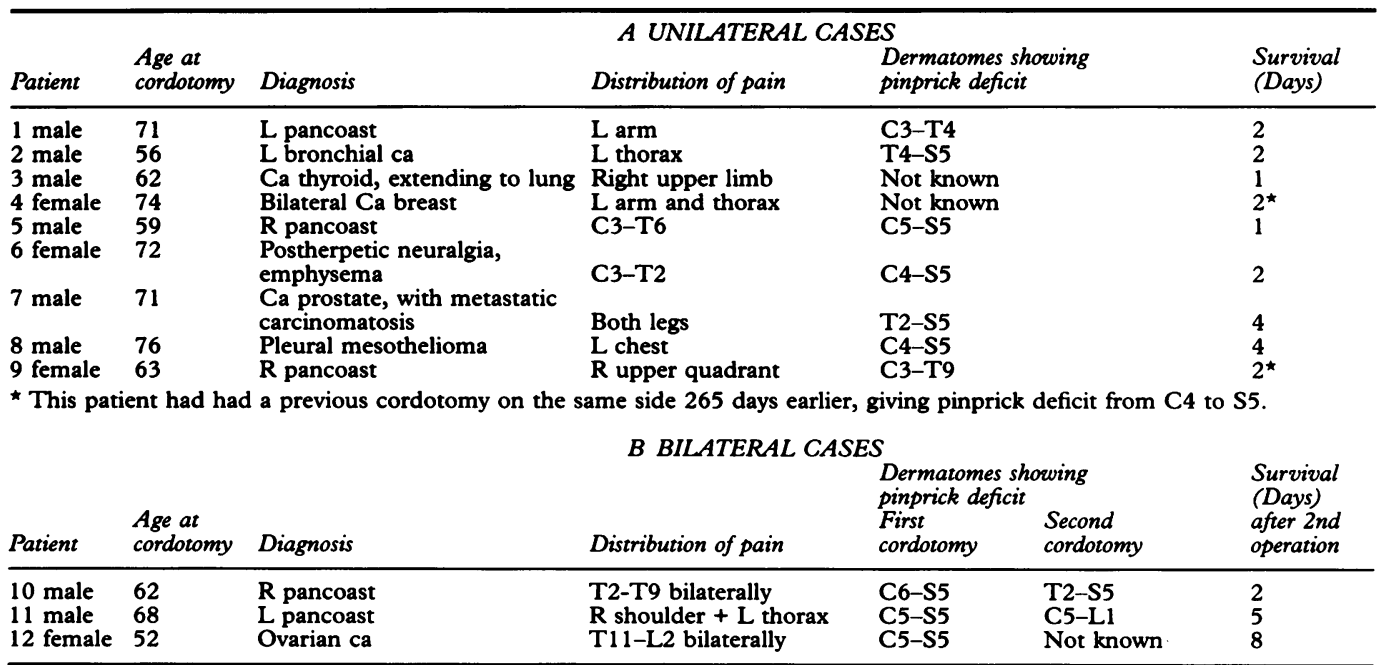


metre grid, whereby the percentage of damaged tissue as a proportion of the whole cord could be calculated.

To compare cords and lesions in different individuals, the planimetrically recorded lesions were transferred to a diagram of the second cervical segment in the atlas of Riley. ${ }^{7}$ Figure 1 shows the schematic diagram used, in which reference points were standardised. The grid pattern (fig 1) enabled us to trace all lesions and other features in every cord onto an identical, and therefore comparable, diagram.

\section{Results}

\section{Clinical}

Respiratory dysfunction appeared very early following uni- or bi-lateral cordotomy in these cases; survival ranged from a few hours to 8 days (table), most dying within 48 hours of operation. Virtually all patients subjected to unilateral cordotomy had pulmonary pathology before operation; and so did two of the bilaterally operated cases. The third patient undergoing bilateral cordotomy had disseminated carcinomatosis. All patients had diaphragmatic and intercostal respiratory movement on the cordotomised side postoperatively, and died in sleep.

The extent of post-cordotomy pinprick deficit is shown in the table for those patients in whom it could be determined. In two unilateral and one bilateral case, the patient's deteriorating condition made it impossible to perform this examination. In the 8 cases in which pinprick deficit was accurately determined, the T4/5 dermatomes were common to every case.

\section{Histological}

Figure 2 is a composite diagram showing the extent of the common lesion at $\mathrm{C} 2$ in all unilaterally- (A) and bi-laterally (B) operated cases. Three unilateral cases $(4,6$, and 7 ) exhibited a bilateral lesion of the anterolateral cord quadrant following operation on one side. Figure 3a shows the preoperative distribution of pain and postoperative pinprick deficit in case 8 ; figure $3 \mathrm{~b}$ is a photograph of the stained

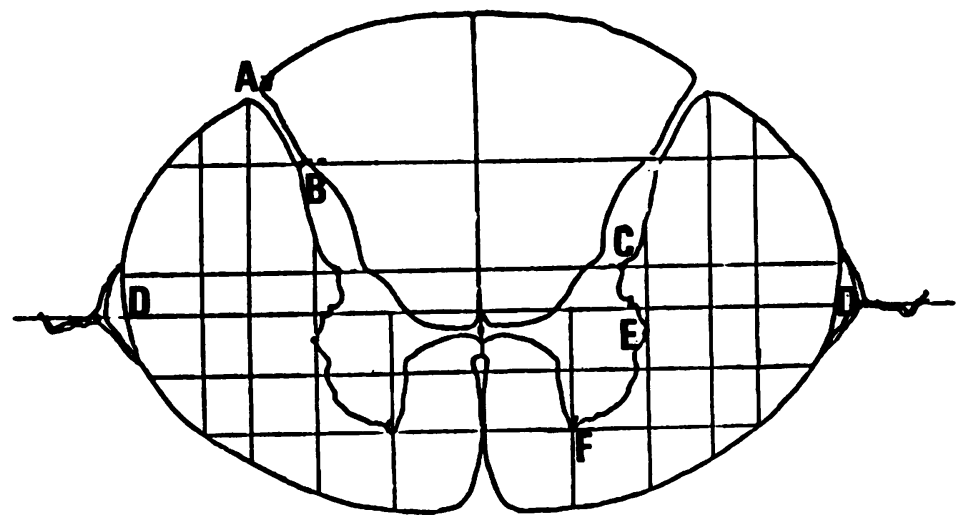

Figure 1 Reference points on transverse section through spinal cord in C2 segment used to allow standardisation of projections: (A) Entry of dorsal root; (B) Apex of dorsal horn; (C) Lateral limit of neck of dorsal horn; (D) Midpoint of attachment of dentate ligament; (E) Most prominent point of lateral border of ventral horn; (F) Anteriormost point of ventral horn. cord, and fig $3 \mathrm{c}$ shows the tracing of the lesion onto a standardised diagram (see fig 1).

Data from our series of 30 cases clinically studied after percutaneous cordotomy at $\mathrm{C} 2$, $^{6}$ in which the cervical cord was anatomically examined, indicates that the stippled area in fig 2 corresponds to the position of ascending fibres representing dermatomes C7-T4; while that in fig 3 corresponds to fibres representing dermatomes T2-L1.

\section{Discussion}

Respiratory dysfunction as a sequel of high cervical cordotomy was first brought to attention by Peet et al. ${ }^{1}$ Since that time, results from large series of cases performed by open surgery $^{23-10}$ or by transcutaneous techni-

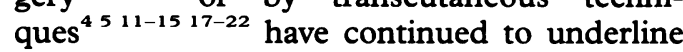
the risk. A study of the literature confirms our experience that apnoea following cervical cordotomy occurs almost exclusively in patients with coexisting pulmonary pathology (particularly neoplasm).

Experimental studies on animals as well as respiratory measurements in humans have been performed to determine the type of respiratory disturbance induced by anterolateral cordotomy. Most authors have emphasised the interruption of descending pathways controlling respiration as the aetiopathological mechanism responsible for this condition. ${ }^{23}$

Krieger and Rosomoff ${ }^{15}$ described 10 patients with respiratory dysfunction syndrome developing within 24-48 hours of cervical cordotomy. They noted that the patients initially manifested a subjective sensation of panic and respiration with frequent sighing before any objective signs became evident. Later, the patients hypoventilated and characteristically exhibited sleep apnoea. If the patient awoke during apnoea, normal respiration resumed, though hypoventilation continued and apnoea recurred if (when) the patient fell asleep again.

A diminution in tidal volume following anterolateral cord lesions 4-5 mm deep was recorded by Belmusto et al $^{11}$ in patients with lung cancer who had cordotomy. The immediate decrease was very profound; and although it recovered within a few minutes, it never regained preoperative values. Nathan ${ }^{16}$ also observed a two thirds reduction in vital capacity following cordotomy in a patient without pulmonary pathology, but noted that respiratory movements on the affected side were not completely eliminated. Both Nathan ${ }^{16}$ and Belmusto et al ${ }^{11}$ observed that the degree of pain relief produced by cordotomy in patients with lung cancer could be predicted from the extent of reduction of tidal volume.

Tenicela et al, ${ }^{4}$ reporting their observations of 41 patients, found that Forced Vital Capacity and Maximum Voluntary Ventilatory Capacity, although reduced preoperatively in patients subsequently developing postoperative dyspnoea, were not significantly changed as a result of cordotomy. On the other hand, Rosomoff et al ${ }^{12}$ observed that the parameter most sensitive to cordotomy was tidal volume. 


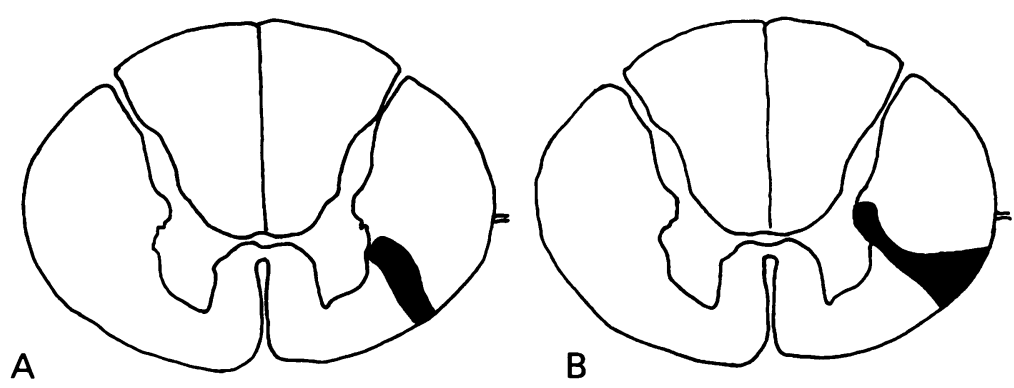

Figure 2 Outlines of lesioned areas at C2 common to all subjects in unilateral cases $(A)$ and following the second operation in bilateral (B) cases.

Its reduction was compensated, when possible, by an increase in respiratory rate. Following bilateral cordotomy, tidal volume was reduced by $36 \%$, and was not compensated by a $32 \%$ increase in respiration rate; but inhalation of $5 \% \mathrm{CO}_{2}$ brought about a return to baseline conditions.

Patients with sleep apnoea, while awake, exhibit both diaphragmatic and intercostal movements; and are able to take a deep(er) breath to command. It may be that sleep apnoea could represent a disturbance of the afferent rather than the efferent control of respiration. The notion that breathing is under "voluntary" control while awake and not while asleep seems to us to involve semantic and perhaps philosophical considerations which cannot be resolved by purely scientific observations.

In experimental work on the dog, Belmusto et $a l^{11}$ found that the spinal pathways controlling respiratory function were intermingled with the "ventral and lateral spinothalamic tracts". As our results on humans indicate, there also seems to be an intimate association between "pain" fibres ascending from upper thoracic segments and fibres controlling ventilation.

The topographical location of these latter fibres in the transverse plane in the $\mathrm{C} 2$ segment (figs 2,3 ) confirms other reports. As a result of his study of eight cases of open high cordotomy, Nathan ${ }^{3}$ produced a diagram illustrating the area common to two cases with respiratory dysfunction and considered to be critical for the control of respiration. Our own composite diagrams (figs 2, 3) defines a smaller area which is included in the dorsal part of Nathan's diagram.

Microphotographs from 3 patients dying of respiratory dysfunction syndrome following bilateral percutaneous cordotomy illustrated by Rosomoff et al ${ }^{12}$ show anterolateral quadrant lesions within the area shown in fig 3 . Tranmer et al $^{21}$ published microphotographs and diagrams of the cords of 3 patients who died of respiratory dysfunction syndrome following cordotomy. Their composite diagram depicting a common lesion area coincides almost exactly with the area deemed critical for the development of respiratory dysfunction in this study.

Our study does give a definite conclusion about the laterality of fibres, damage to which gives rise to fatal respiratory dysfunction syndrome, as in most instances a bilateral cord lesion was found. In those cases in which the cord lesion was strictly unilateral, it was on the side opposite the lung with abnormal preoperative function. This indicates that the lesion would affect a pathway subserving the ipsilateral lung (shown at necropsy to be unaffected in most cases), responsible for maintaining adequate ventilation in these patients. However, the likelihood of the existence of a bilateral pathway, the lesion of which produces respiratory dysfunction syndrome, is supported by the absence of this syndrome after unilateral cordotomy in patients with no pulmonary pathology, and its occurrence in the same patients after bilateral procedure.

Why do most patients survive after bilateral high cervical cordotomy? We agree with one of Nathan's ${ }^{16}$ theories that the critical area is not damaged on at least one side, with a concomitantly low level of pinprick deficit on that side. Tenicela et $a l^{4}$ described 10 patients who apparently did not develop respiratory dysfunction syndrome after bilateral cervical cordotomy; eight had an upper level of analgesia below T4 on one side.

What is the nature of the neural messages conveyed by the pathway in the anterolateral quadrant which is destroyed by cordotomy at C2 to produce respiratory dysfunction syndrome? It is well recognised that impulses from both low- and high-threshold receptors ascend in the anterolateral quadrant; ${ }^{25}$ but it is not known for certain whether respiratory afferents are included in this pathway. Remmers, ${ }^{26}$ however, showed that proprioceptive information from intercostal muscles and tendons in cats reaches supraspinal levels. Another possibility is that the responsible anterolateral fibres carry information from broncho-pulmonary afferents. Such information is bilaterally represented in the nucleus of the solitary tract, with approximately one third coming from the contralateral side. This has also been described in cats. ${ }^{27}$ These messages travel peripherally in the vagus nerve. However, Adams and Victor ${ }^{28}$ suggest that in humans some afferents may enter the spinal cord via sympathetic rather than parasympathetic peripheral afferents; and in the cat, Foreman et al ${ }^{29}$ elicited activity in ascending anterolateral axons of spinothalamic and spinoreticular pathways by stimulating the sympathetic chain between the 2 nd and $3 \mathrm{rd}$ thoracic ganglia, as did Ammons et $a l^{30}$ by stimulating the stellate ganglion. It is thus relevant that sympathetic afferents from the lungs and bronchi enter the cord between $\mathrm{T} 2$ and $T 7,{ }^{31}$ and that the "pain fibres" representing these segments are precisely those whose destruction is critical for the development of the respiratory dysfunction syndrome. The vast majority of fibres ascending in the anterolateral funiculus of the spinal cord which are destroyed by cordotomy are spinoreticular, ${ }^{32}$ and could be expected to participate in the arousal mechanism, including arousal of brainstem respiratory centres. ${ }^{15}$ These mechanisms are greatly attenuated, if not dormant, during sleep but reactivated during wakefulness.

The clinical and anatomical results of this study clearly indicate that the fibres whose 

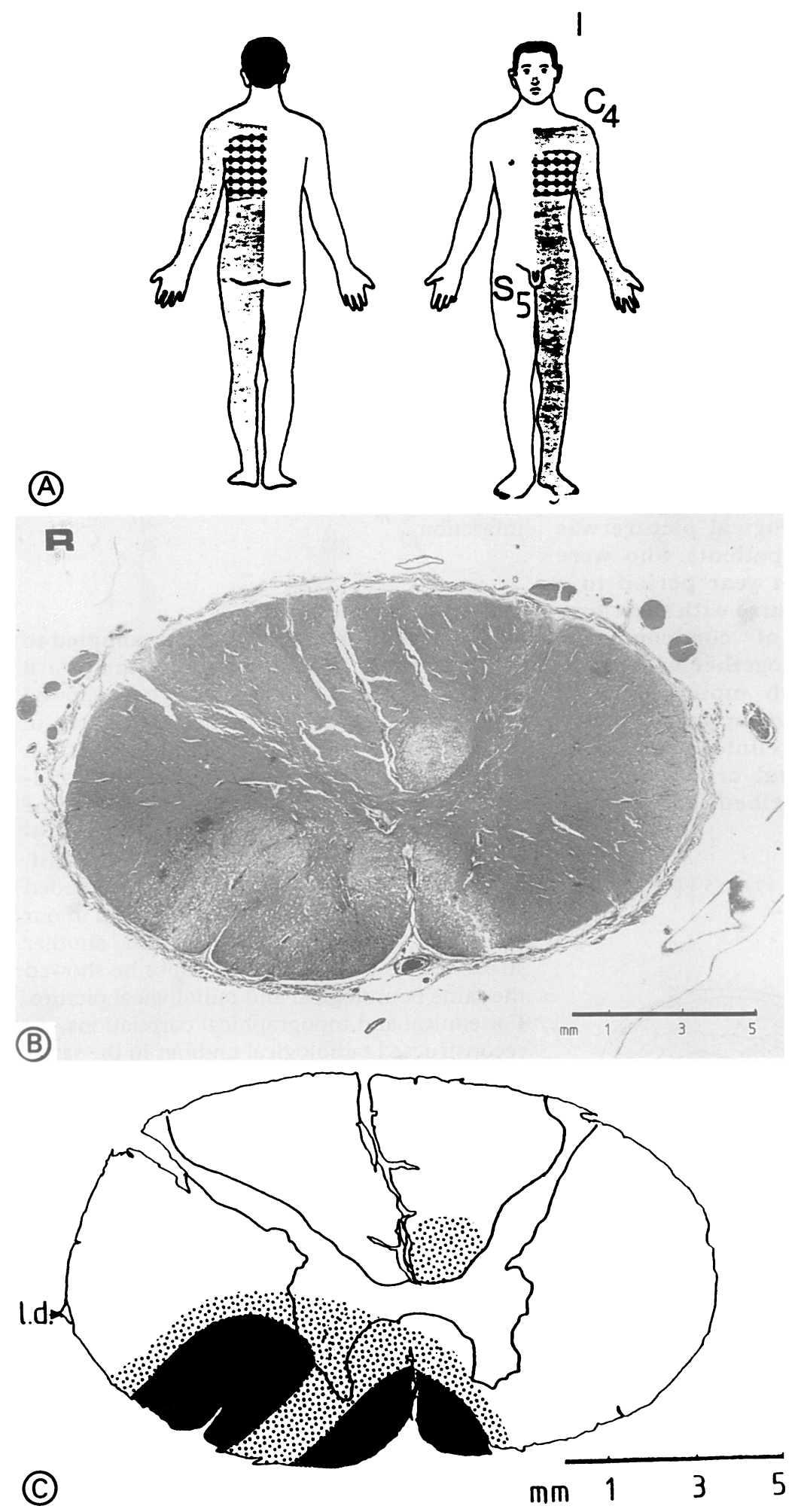

Figure 3 Case 8, male age 76 with bilateral asbestosis and left pleural mesothelioma; pain in left chest; cordotomy resulted in complete relief of pain, with absence of pinprick sensation from C4 to S5. postoperative survival 4 days: (A) Postoperative clinical diagram, showing region of original pain (crosses) and region of pinprick loss following cordotomy (shaded); (B) $15 \mu \mathrm{m}$ paraffin section through $C 2$ cord segment, stained with Holmes' Luxol Fast Blue, $(R=$ right); (C) Standardised projection drawing of $B$ (see fig 1).

destruction is responsible for the appearance of respiratory dysfunction syndrome are intimately related to fibres of the "pain pathway", particularly those representing the T2-T5 segments. While there is no proof that they are afferent in nature, we feel that this possibility should not be entirely dismissed.
We are grateful to the Cancer Relief Campaign without whose support this work could not have been undertaken.

1 Peet MM, Kahn EA, Allen SS. Bilateral cervical chordotomy for relief of pain in chronic infectious arthritis. omy for relief of pain

2 Ogle WS, French LA, Peyton WT. Experiences with high cervical cordotomy. $\mathcal{F}$ Neurosurg 1956;13:81-87.

3 Nathan PW. Results of antero-lateral cordotomy for pain in cancer. F Neurol Neurosurg Psychiatry 1963;26:353-62.

4 Tenicela F, Rosomoff HL, Feist J, Safar P. Pulmonary function following percutaneous cervical cordotomy. Anaesthesiol 1968;29:7-16.

5 Mullan S, HosobuchiY. Respiratory hazards of high cervical percutaneous cordotomy. $\mathcal{f}$ Neurosurg 1968; 22:291-7.

6 Lahuerta J. El funiculo anterolateral de la médula espinal humana en la nocicepción y somestesia. Estudio anatomoclinico y somatosensorial cuantitativo basado en la cordotomia cervical percutánea. Thesis, University of cordotomia cervic

7 Riley HA. An atlas of the basal ganglia, brain stem and spinal cord based on myelin-stained material. Baltimore: Williams and Wilkins, 1943.

8 Brihaye J, Rétif J. Comparaison des résultats obtenus par la cordotomie antéro-latérale au niveau dorsal, et au niveau cervical. Neurochirurgie 1961;7:258-77.

9 Schwartz HG. High cervical cordotomy-technique and results. Clin Neurosurg 1962;8:282-93.

10 White JC, Sweet WH. Anterolateral cordotomy: Open versus closed - comparison of end results. Adv Pain Res Therap 1979;3:911-19.

11 Belmusto L, Brown E, Owens G. Clinical observations on respiration and vasomotor disturbance as related to respiration and vasomotor disturbance as related

12 Rosomoff HL, Krieger AJ, Kuperman AS. Effects of percutaneous cervical cordotomy on pulmonary function percutaneous cervical cordo

13 Rosomoff HL. Bilateral percutaneous cervical radiofrequency cordotomy. I Neurosurg 1969;31:41-46.

14 Fox JL. Localization of the respiratory motor pathway in the upper cervical spinal cord following percutaneous cordotomy. Neurol 1969;19:1115-18.

15 Krieger AJ, Rosomoff HL. Sleep-induced apnoeas. I. A respiratory and autonomic dysfunction syndrome following bilaterial percutaneous cervical cordotomy. 7 Neurosurg 1974;39:168-80.

16 Nathan PW. The descending respiratory pathway in man. $f$ Neurol Neurosurg Psychiatry 1963;26:487-99.

17 Krieger AJ. Sleep apnea produced by cervical cordotomy and other neurosurgical lesions in man. In: C Guillemiand other neurosurgical lesions in man. In: C Guilleminault, Dement $\mathrm{B}$ Liss, 1978:273-94.

18 Tasker RR. Percutaneous cordotomy - the lateral high cervical technique. In: Schmidek HH, Sweet WH, eds. Operative neurosurgical techniques, vol 2. New York: Grune and Stratton, 1982:1187-53.

19 Chevrolet J-C, Reverdin A, Suter P, Tschopp JM, Junod AF Ventilatory disturbance resulting from bilateral anterolateral high cervical cordotomy. Dual beneficial effect of aminophylline. Chest 1983;84:113-15.

20 Ischia S, Ischia A, Luzzani A, Toscano D, Steele E. Result up to death in the treatment of persistent cervico-thoracic (Pancoast) and thoracic malignant pain by unilateral cervical cordotomy. Pain 1985;21:339-55.

21 Tranmer BI, Tucker WS, Bilbao JM. Sleep apnea following percutaneous cervical cordotomy. Canad 7 Neurol Sci 1987;14:262-7.

22 Lipton S. Percutaneous cordotomy. In: Wall PD, Melzack R, eds. Textbook of pain. Edinburgh: Churchill Livingstone, 1989:832-9

23 Hitchcock ER, Leece B. Somatotopic representation of the respiratory pathways in the cervical cord of man. $f$ Neurosurg 1967;27:320-9.

24 Belmusto L, Woldring S, Owens G. Localization and patterns of potentials of the respiratory pathway in the cervical spinal cord in the dog. $f$ Neurosurg 1965;22 277-83.

25 Noordenbos W, Wall PD. Diverse sensory functions with an almost totally divided spinal cord. A case of spinal cord transection with preservation of one anterolateral quadtransection with preservation

26 Remmers JE. Extra-segmental reflexes derived from intercostal afferents: phrenic and laryngeal responses. $\mathcal{F}$ Physiol costal afferents: phrenic and

27 Kalia M, Mesulam MM. Brain stem projections of sensory and motor components of the vagus complex in the cat III. Laryngeal, tracheobronchial, pulmonary, cardiac, and gastrointestinal branches. $f$ comp Neurol 1980;193 467-508.

28 Adams RD, Victor M. Principles of neurology, 4th ed. New York: McGraw Hill, 1989.

29 Foreman RD, Blair RW, Weber RN. Viscerosomatic convergence onto $T_{2}-T_{4}$ spinoreticular, spinoreticular-spinothalamic, and spinothalamic tract neurons in the cat. Exp Neurol 1984;85:597-619.

30 Ammons WS, Girardot $M N$, Foreman RD. $T_{2}-T_{\text {, spinotha- }}$ lamic neurons projecting to medial thalamus with viscerosomatic input. 7 Neurophysiol 1985;54, 73-89.

31 Johnson RH, Spalding JMK. Disorders of the autonomic nervous system. (Contemporary neurology series). Philadelphia: F A Davies, 1974.

32 Bowsher $D$. Les relais des sensibilitiés somesthésique et douloureuse au niveau du tronc cérébral et du thalamus. Toulouse Méd 1963;64:965-84. 